\title{
La verdad como valor integrador desde el pensamiento complejo de Santo Tomas de Aquino
}

\section{The truth as an integrating value from the complex thought of Santo Tomas de Aquino}

Andrzej Lukomski, Ph.D.

Universidad de La Salle, Colombia

Autor para correspondencia: gavilondocarlos@ gmail.com

Fecha de recepción: 29 de Junio de 2016 - Fecha de aceptación: 05 de Julio de 2016

\section{Resumen}

Esta ponencia presenta es avance del Proyecto titulado Comprensiones Y Prácticas De La Formación Integral. Mi intención es presentar la verdad como el valor de gran importancia en la clave del pensamiento complejo de Santo Tomás de Aquino. Con esta propuesta se pretende presentar una lectura de Santo Tomas que reviva el papel de pensar de manera unificadora los problemas éticos, filosóficos, proyectos educativos, y aportes desde el pensamiento religioso para construir la persona íntegra, comprometida con los problemas de este mundo y abierta a la transcendencia la cual no es apartada de lo real.

Palabras claves: verdad; valor; formación integral; pedagogía; prácticas pedagógicas; experiencia educativa

\begin{abstract}
This is an advance of the Project entitled: Comprehensions and Practices of the Integral Formation. My intention is to present the truth as the value of great importance in the key of the complex thought of St Tomas de Aquino. With this offer one tries to present a Reading of St Tomas who re-lives the paper of thinking, through a unifying way, about the ethical, philosophical problems, educational projects and the contributions from the religious thought, to construct the outstanding individual copromised with the problems of this world and opened for the transcendence which is not left out of the real.
\end{abstract}

Key words: truth; value; integral formation; pedagogy; pedagogic practices; educational experience 


\section{Introducción}

En la descripción bíblica de la creación del hombre podemos leer que Dios creo al hombre como hombre y mujer. El hombre es acompañado por la mujer. De esta descripción podemos sacar cierta analogía en el campo de los diferentes saberes. Un saber disciplinario no puede ser solo debe estar acompañado por otro. El acompañamiento en la educación está vinculado con la formación integral. En el campo de los diferentes saberes. Podemos hablar sobre multidisciplinariedad. Los saberes disciplinares no pueden renunciar del acompañamiento de los saberes éticos, filosóficos, teológicos, para que estos saberes no pierdan el fin para el cual son subordinados que es el bien del hombre, el mejoramiento de su vida y el reconocimiento su dignidad.

\section{Los desvíos modernos en la comprensión de la verdad}

En mi opinión personal, el pensamiento de Santo Tomás puede proporcionar ideas revitalizantes y permitir despedirse con las propuestas que nos ofreció la modernidad, un experimento valioso para la mente humana pero demasiado reduccionista, elitista y ciego frente a lo que significa el mundo global y toda la riqueza del pensar que no necesita ser orientada por la dominación del pensamiento occidental y sus propuestas.

Lo característico de la modernidad fue tener relatos completos. Ejemplo de esto es el idealismo alemán con su figura más representativa de Hegel. Este filósofo tenía pretensión construir un relato de todo y sobre todo donde la última justificación y legitimización se encontraba en la verdad de la razón humana como portadora de la verdad absoluta. Esos fines los podemos ver en su Fenomenología del Espíritu. La lectura de Hegel en mi opinión provoca la pregunta: ¿podemos la verdad de la razón humana considerar como absoluta? Si las cosas fueron así, cada ser humano puede considerar que tiene la verdad absoluta. De esta manera tenemos tantas verdades absolutas como los seres humanos o ninguna verdad es absoluta o simplemente no hay verdad, todo es relativo. Esta conclusión no me dejaba en paz interior. Y para mí fue algo revelador mirar la propuesta de Santo Tomás que propone otro referente para la verdad que es Dios. "Yo soy la verdad"( J. 14:6) Así se determina Jesucristo verdadero Dios y hombre como lo presenta la fe cristiana.

\section{Dios como referente de la verdad, una propuesta alternativa frente la modernidad}

Cuando hice cierta lectura de la Suma Teológica mirando en ella el problema de la verdad, pude notar que directamente a este problema se dirige la cuestión 16. No obstante en toda Suma Teológica donde Santo Tomás plantea cerca de dos mil preguntas en la respuesta de cada una de ellas está el deseo de encontrar la verdad. No hay pretensión de Santo Tomás de decir todo sobre la verdad, no hay pretensión de decir que todo lo que él dijo contiene la verdad en plenitud. En santo Tomás tenemos reconocimiento de la existencia de Dios y Él es referente fundamental en el problema de la verdad a diferencia de los pensadores modernos que ubican la verdad únicamente en el entendimiento humano.

En santo Tomás la verdad es identificada con el entendimiento de Dios y con ello con el mismo Dios. El entendimiento humano tiende hacia la verdad y en el entendimiento humano la verdad se presenta siempre como adecuación. La adecuación es algo que se requiere de manera absoluta pero esta adecuación no es absolutamente dada. El conocimiento de la verdad no es dado de manera innata. Cuando se separa el problema de la verdad de la existencia de Dios, a mi 
modo de ver pasa lo que pasa con una flor cuando se la corta de su raíz y se mete en un florero. Esa bonita flor metida en un florero por algún rato conserva su belleza pero en algún tiempo se está marchitando, pues la verdad desde la razón humana pierde su belleza y su atractivo en algún momento.

Este proceso donde la verdad pierde la belleza podemos observarlo a través de algunos filósofos modernos en su reflexión acerca de la verdad. Descartes ("Discurso del Método") cuando hace alguna mención sobre verdad se refiere a las ideas claras y distintas, para Kant (“Critica de la razón pura") la verdad está vinculada con las condiciones a priori de nuestra experiencia. Para Hegel ("Fenomenología de Espíritu) la síntesis es la que asume toda la diferencia.

Desde Marx ("La miseria de la Filosofía") el problema de la verdad comienza a marchitarse por estar vinculado con el interés de las clases dominantes. Aquí el problema de la verdad se ve bajo manipulación. Si el problema de la verdad es el problema de la razón aquí aparece la pregunta de qué razón. La respuesta de Marx es la razón de la clase dominante la cual busca justificar su forma de actuar.

Nitzsche ve en la verdad algo que incrementa el sentimiento de poder. Cfr .Nitzsche, F .Nagelasseme Fragmente, Herbst 1887,9 [91] . en Werke cit., 8,2 1970, lin 4-19:

Alarcón nota que la palabra verdad puede ser cuestión de cierto juego. La palabra verdad puede significar todo lo que dice un personaje llamado gurú. De acuerdo con este juego nosotros tanto tiempo estaremos en el campo de juego hasta donde reconozcamos las palabras de gurú como verdaderas. En el juego de ajedrez los movimientos de cada figura son determinados por el juego. Cuando el rey queda amenazado de tal manera que no tiene ninguna salida el juego se acaba. Cuando al gurú se presenta alguna falsedad el juego es acabado. Alarcon (2000:36)

La verdad dentro del juego logra algo grotesco y de este modo el problema de la verdad pierde toda su magnitud e importancia, vienen otros términos que la desplazan o subordinan como por ejemplo el poder, los intereses de clase, el éxito, por dar algún ejemplo. El problema de la verdad podemos también cambiarlo en cierto tecnicismo lógico. La verdad puede ser analizada desde este formalismo lógico para su criterio de validez.

En este momento quisiera expresar mi posición propia la cual pienso que identifico con la propuesta de Santo Tomás, si excluimos a Dios como referente de la verdad no encontramos ningún referente serio que nos permita ver la verdad en toda su dimensión real solamente podemos reducirla a un conjunto de ideas de una degradación progresiva. Así llegamos a esto que la verdad es esto lo que uno quiera que sea.

Para santo Tomas como lo expresa en q.38 a.1 "...la verdad y la bondad divina son el principio del conocer..." Santo Tomas (2001:374)

$\checkmark$ La verdad en el entendimiento divino es absoluta y no mutable.

$\checkmark$ ¿Cómo encontramos el problema de la verdad en el entendimiento humano?

$\checkmark$ En artículo 8 de la cuestión 16 Santo Tomás pregunta: “¿La verdad es o no es inmutable?" 
La respuesta es la siguiente: ... propiamente la verdad está en el entendimiento, y las cosas son llamadas verdaderas por la verdad que hay en algún entendimiento .Por lo tanto la mutabilidad de la verdad hay que analizarla con respecto al entendimiento, cuya verdad consiste en que tenga conformidad con las cosas conocidas. Y dicha conformidad puede cambiar en dos maneras (...) Una manera es por parte del entendimiento que se tenga de una u otra opinión de parte de la misma cosa. La otra manera si manteniendo la misma opinión de una cosa, la cosa no cambie. Por lo tanto hay algún entendimiento en el que no puede darse un cambio de opinión, o al que no se escape nada, en el la verdad es inmutable (...) un entendimiento así lo es el divino. Por eso la verdad de entendimiento divino es inmutable. En cambio la verdad de nuestro entendimiento es cambiable.

No porque este sometida a la mutación, sino porque nuestro entendimiento pasa de la verdad a la falsedad. Santo Tomás (2001:231)

Santo Tomás no niega que cada ser humano tiene su propio entendimiento. Aquí se distancia de Aristóteles y de sus interpretadores del mundo árabe, cómo Avicena donde se considera que el entendimiento de cada persona es solo una participación en algún entendimiento universal no personal.

El entendimiento es, como lo dijera Zubirii ("Inteligencia sentiente" o "Inteligencia y Logos) de cada suyo. Este entendimiento en el camino hacia verdad pasa por diferentes procesos, enriquecido por diferentes instrumentos no solo teóricos sino científicos; los laboratorios, microscopios, telescopios, diferentes instrumentos de medir que pueden potencializar sus capacidades. Así la ecuación de entendimiento con la cosa no sólo se funda en ecuación de nuestro ojo, no solo conocidas según los datos logrados por observación ocular sino también por observación microscópica o macroscópica de los objetos del entendimiento.

Por otra parte Santo Tomás no identifica la mutalidad de la verdad con la relatividad, lo que podemos apreciar en las siguientes confirmaciones expresadas en el artículo 8 de la cuestión 16: "...la verdad cambia no porque en ella no permanezca ninguna verdad, sino no permanece la verdad que era antes." Santo Tomás (2001: 231)

La proposición no solo contiene la verdad como otras cosas de las que se dice que la contienen en cuanto cumplen lo que esta ordenado por el entendimiento divino, sino que contiene la verdad de un modo especial, en cuanto expresa la verdad del entendimiento, la cual consiste en la conformidad entre el entendimiento y la cosa. Anulada dicha conformidad cambia la verdad de la opinión y consecuentemente la verdad de la proposición. (2001: 232)

En artículo 1 de la cuestión 20 Santo Tomás precisa a qué tipo de verdad está orientado el entendimiento: “...el entendimiento está orientado antes a la verdad general que a las verdades particulares." Santo Tomas (2001:258)

Al final en q. 64 a.1 santo Tomás aclara: Hay dos clases de conocimiento de la verdad. Uno se obtiene por la gracia. Otro por la naturaleza. El que se obtiene por la gracia, a su vez, se divide en otros dos: uno que es solamente especulativo, como el de aquel a quien se le revela 
algún secreto divino. Otro que es afectivo y produce el amor de Dios. Este es que propiamente pertenece al don de sabiduría. Santo Tomas (2001:593)

Profundizando esta idea en q.60 a.1 podemos leer: "La verdad del conocimiento natural es distinta de la verdad de conocimiento infuso o adquirido." Santo Tomas (2001:560)

Esta idea el expreso también Galileo cuando dijo que hay cierta diferencia entre conocer cómo se va al cielo y cómo va el cielo. Reale (2004: 364)

De aquí pienso que vale la pena conservar las proporciones. El conocimiento obtenido por la gracia no se puede confundir con el conocimiento natural. En mi opinión el conocimiento obtenido por la gracia es un conocimiento que podemos darle el valor absoluto, el conocimiento natural es un conocimiento perfectible con la característica de mutabilidad. Obviamente hablamos sobre conocimiento de verdad y no LA VERDAD en su presencia absoluta.

En opinión de Santo Tomás (q.64 a.2) conocer la verdad natural en diferencia de la verdad sobre algo transcendente es la cuestión de perspicacia del entendimiento aprovechando la luz natural del intelecto. Santo Tomas (2001:594)

\section{El criterio de la verdad según Santo Tomás}

En artículo 6 de cuestión 16 Santo Tomás pregunta:” ¿Hay o no hay una sola verdad como criterio de todo lo verdadero?"

La respuesta a esta pregunta es la siguiente: “...una sola es la verdad del entendimiento divino, según la cual todas las cosas son llamadas verdaderas.”

Y en artículo 8 de la misma cuestión 16 podemos leer “...la verdad del entendimiento divino, el criterio de lo que todo lo demás sea o no sea verdadero.” Santo Tomás (2001: 231)

Si el criterio de la verdad es la verdad que está en el entendimiento divino, entonces existe la verdad como el referente para lo verdadero de las cosas. Desde esta perspectiva el entendimiento humano en cierto sentido es el criterio de verdad y en cierto sentido no lo es. El entendimiento humano como creado por Dios tiene en si los principios de la verdad y en este sentido es critérium de la verdad sin embargo el entendimiento de cada persona tiene sus propias capacidades y desde esas capacidades existen diferentes verdades. Esas verdades personales por este elemento personal no pueden ser critérium de la verdad como tal. Sin embargo esta verdad de entendimiento individual no es verdad subjetiva y relativa. La verdad del entendimiento individual entiendo como verdad objetiva de acuerdo con las capacidades de entendimiento. El entendimiento divino es el criterio de la verdad en términos absolutos. Desde el entendimiento divino algo es absolutamente verdadero y yo me atrevería a decir solo desde este entendimiento.

Si se trata de nuestro entendimiento santo Tomás en artículo 7 de cuestión 16 dice: "Nuestro entendimiento no es eterno, tampoco lo es la verdad de los enunciados formados por nosotros "Santo Tomas (2001:231). Nuestro entendimiento no es la mera de todas las cosas pero en nuestro entendimiento exista tan mera por el hecho de que fuimos creado por Dios y desde aquí en nuestro entendimiento existen principios desde las cuales se establece la conformidad de 
la cosa con el principio. El Círculo de Viena como principio de verdad reconoció la verificación. Esta verificación tenía sus procedimientos.

Otra propuesta para entender la verdad es el criterio de falsación. La falsación no permite reconocer que alguna proposición es falsa hasta cuando no se demuestra los procedimientos que la pueden falsificar. Desde aquí Popper dice que por ejemplo tal sistema filosófico como marxismo nunca puede ser falsificado por eso no se puede definir si es verdadero o es falso la situación parecida es propia según este autor al psicoanálisis de Freud .Popper (2010: 407)

En mi opinión todos los sistemas filosóficos que conocemos no disponen de procedimiento de falsación por el cual no se puede definir que son verdaderos o falsos de manera absoluta. Escoger tal o cual sistema como referente en el análisis de la realidad es cuestión de entendimiento de la comunidad científica o tradición filosófica con la cual uno se quiere identificar.

\section{La condición del ser humano y el problema de la verdad}

En la cuestión 31 articulo 1 podemos leer que: "Propiamente, el pensamiento consiste en la búsqueda de la verdad: búsqueda que en Dios no se da, puesto que cuando el entendimiento ha alcanzado la forma de la verdad, no piensa sino que contempla perfectamente la verdad." Santo Tomás 2001: 356)

Pienso que esta distinción es muy importante porque pensar la verdad y contemplar son cosas distintas. Pensar implica la continua búsqueda, la aproximación pero nunca lo que está en nuestro entendimiento corresponde de forma completa y agotable de la plena verdad. Tener en nuestro entendimiento la relación de igualdad con lo que se conoce es algo de nuestros deseos que en nuestra vida terrenal no es posible realizar plenamente. Con esto no quiero negar la posibilidad de conocer la verdad sino expresar la posición de la imposibilidad de tener la verdad sobre lo conocido de modo absoluto y pleno tal como esto es posible en el entendimiento divino. El entendimiento creado no tiene esta capacidad que el entendimiento no creado.

En cuestión 16 articulo 2 Santo Tomas escribe :"..El entendimiento puede conocer la adecuación existente entre él y lo conocido pero no la aprehende de aquello lo que es..."Santo Tomás (2001:260). Entiendo esto como la adecuación de entendimiento con algo abstraído de la realidad y no con la realidad misma.

En cuestión 76 artículo 5 se propone ver la posición del hombre desde orden natural:...el alma intelectiva (...)en el orden de la naturaleza ocupa lugar el más bajo lugar entre las sustancias intelectuales, debido a que no le es connatural el conocimiento innato de la verdad como si lo es en los ángeles, sino que se ve obligada a desgranarla a través de los sentidos tomándola de la multiplicidad de las cosas . Santo Tomás (2001:694). En forma de cierta ironía podemos decir que en la modernidad parece que el hombre logro la fe de poseer el intelecto angelical es decir el hombre comenzó a creer que es posible lograr por sus propias capacidades el conocimiento sin engaño y falsedad.

Santo Tomás ubica el hombre dentro de orden creado tal como se presenta desde la Sagrada Escritura. En la construcción de nuevos relatos en mi opinión tiene profundo sentido 
reencontrarse con las meditaciones de santo Tomas no para restaurar el pensamiento moderno sino para construir alguna alternativa para este pensamiento. Santo Tomás está muy lejos de soluciones simples, de reduccionismo de respuestas que apelan solo a alguna forma limitada de ver la verdad. Esta forma limitada la podemos llamar ideologías. El problema de la verdad no es cuestión de ideología es el problema de la realidad, es el problema de nuestro ser.

A manera de conclusión: la verdad como una sinfonía de ser: Desde mi lectura de Santo Tomas veo su forma de mirar la creación de Dios como una gran obra de arte. Como una sinfonía. En una sinfonía hay una multitud de instrumentos, multitud de voces. Estos instrumentos tienen su propia composición. Son los instrumentos que cada uno por su parte puede funcionar independiente de otro. Sin embargo todos estos instrumentos pueden estar juntos y a pesar de todas sus diferencias puede unirse en una sola melodía pueden ser una unidad múltiple, multiforme unida en una sola voz. Así veo el universo creado por Dios y el esfuerzo de encuentros multidisciplinares donde se crean unidades emergentes. De esta visión de universo sale también propuesta para la formación integral, desde la cual cada persona debe reconocer su propia complejidad, como la complejidad de mundo y desde aquí crear su propia sinfonía donde el papel de educador es ser acompañante en esta autoconstrucción.

\section{Bibliografía}

Alarcón, E, El debate sobre la verdad, en P. Pérez-Ilzarbe, R. Lázaro (eds.), Verdad, bien y belleza (Cuadernos de Anuario Filosófico. Serie Universitaria 103, Pamplona, 2000) 35-62.

Aristóteles. Acerca del alma. (2004) Losada. Buenos Aires

Aristóteles. Metafísica. (2000) Gredos. Madrid

Descartes, R. Discurso del método. (2001) Libsa. Madrid

Feuerbach, L. La Esencia de cristianismo. (1975) Sígueme. Salamanca. España

Kant,E. Critica de la razón pura. (2007) Losada. Buenos Aires

Lukomski, Andrzej. La verdad como valor integrador desde el pensamiento complejo de Santo Tomas de Aquino. Profesor Titular Universidad de la Salle. Bogotá, Colombia. ajurczynski@unisalle.edu.co

Marks. K. Miseria de la filosofía (2005) Libros de bolsillo. Buenos Aires

Nitzsche , F .Nagelasseme Fragmente, Herbst 1887 ,9 [91] . en Werke cit., 8,2 1970, lin 4-19:

Popper,K. La sociedad abierta y susenemigos(2010) Paidos. Barcelona

Reale. G. Historia del pensamiento filosófico y científico T. II. (2004). Herder. Mèxico. 1985) Revista de Occidente. Madrid

Santo Tomas. Suma Teológica. (2001). Biblioteca de Autores Cristianos. Madrid. España

Zubiri. J. Inteligencia sentiente. (2006) Alianza Editorial Madrid

Zubirii .j Inteligencia y Logos (1982) Alianza Editorial. M Madrid 